gives experts a bigger role in decision making based on professional psychiatric assessment, without compromising the rights of the patient. It establishes an independent mental health tribunal, a national mental health coordination committee and district mental health coordinating committees. The role of the judiciary and the police is clearly defined. The passing of this Bill will greatly enhance psychiatric care and safeguard the rights of people who are mentally ill.

\section{Conclusions}

- U ganda is a low-income country that has recently begun to emerge from decades of civil strife and wars.

- It has a high level of poverty and low literacy rates, as well as a high proportion of young people.

o There is a significant burden of mental health problems, worsened by the psychosocial effects of civil wars and the HIV/AIDS pandemic.

O Health reforms in the past 5 years or so, together with formulation of the national mental health policy, have led to a sizeable investment in education and training as well as infrastructure in the mental health sector.

o The challenges that remain concern: the integration of mental health into primary healthcare, which has to overcome some resistance and issues of stigma; the inadequate number of specialists (psychiatrists, clinical psychologists, psychiatric social workers); the limited availability of newer psychiatric drugs; mental health promotion and prevention; improved education and training in psychiatry; and the limited awareness of mental health consumers of their rights.

\section{References and further reading}

Boardman, J. \& O vuga, E. B. L. (1997) Rebuilding psychiatry in U ganda. Psychiatric Bulletin, 21, 649-655.

Bolton, P., Bass, J., Neugebauer, R., et al (2003) Group interpersonal psychotherapy for depression in rural U ganda: a randomized controlled trial. Journal of the American Medical Association, 289, 3117-3124.

Kigozi, F., Kinyanda, E. \& Kasirye, R. (1999) Street children in Uganda: a product and a high risk group for HIV/AIDS. Southern African Journal of Child and Adolescent Mental Health, 2.

Kinyanda, E. (1998) Frequency with which psychiatric disorder is associated with a positive HIV-1 serostatus as seen in persons attending a TASO clinic in Mulago. South Africa Medical Journal, 88, 1178 .

Kinyanda, E. \& Musisi, S. (2001) War traumatisation and its psychological consequences on the women of Gulu district. In Medical Interventional Study of War Affected Gulu District, U ganda. Kampala: ISIS-WICCE.

Kinyanda, E., Hejelmeland, H. \& Musisi, S. (2004) Deliberate self-harm as seen in Kampala, U ganda: a case-control study. Social Psychiatry and Psychiatric Epidemiology, 39, 318-325.

Musisi, S. \& Kinyanda, E. (2000) Psychiatric Problems of HIV/ AIDS and Their Management in U ganda. A Book for Primary Health Care Workers. Kampala: STIP/Ministry of Health.

Musisi, S., Kinyanda, E., Leibling, H., et al (1998) The psychological consequences of war traumatisation on women of Luwero district, U ganda. In Short Term Intervention of the Psychological and Gynaecological Consequences of Armed Conflict in Luwero District. Kampala: ISIS-W ICCE.

Musisi, S., Tugumisirize, J., Kinyanda, E., et al (2001) Psychiatric consultation liaison at Mulago Hospital, Kampala U ganda. Makerere University Medical School Journal, 35, 4-11.

UNAIDS (2004) 4th Report on the Global AIDS Epidemic. Geneva: UNAIDS.
This paper was presented at the annual meeting of the Royal College of Psychiatrists, Harrogate, UK, 2004.

\title{
Some cultural aspects of the Arab patient-doctor relationship
}

\section{Fakhr El-Islam}

Academic Consultant, Behman Hospital, Helwan, Cairo, Egypt, email: info@behman.com.

ulture is a socially shared, trans-generationally transmitted system of implicit values, beliefs and attitudes and explicit behavioural practices (Kroeber \& Kluckhohn, 1952). It includes religion. Culturally based assumptions infiltrate the patientdoctor relationship.

\section{The doctor as the 'other'}

Patients transfer to doctors culture-based attitudes related to the doctor's gender and age. A female patient may avoid eye contact with a male doctor because this is culturally polite and respectful. A male patient may not report any weakness (e.g. fears, tearfulness or sexual inadequacy) to a female doctor. During family therapy for intergenerational conflict (El-Islam et al, 1986), children are likely to expect the doctor to have the same attitudes as their parents, and members of the parental generation are often surprised when the doctor does not oppose children's attempts to depart from their cultural heritage. Good doctors are generally expected to be authoritarian to some extent and their instructions directly advisory rather than a choice or an invitation to reflect (e.g. about alternative methods of treatment). Patients expect doctors to take their side in conflicts with family members, employers and public authorities. 
worried about the risk of drug dependence. They are likely to ask for psychological treatments, which are considered safer and more radical in dealing with the root cause of their illness. However, the majority of Arab patients have much less confidence in psychological or 'talking' therapies than in physical therapies.

During individual psychotherapy, it is advisable to punctuate individual sessions with Arab patients by joint sessions with key family members. This avoids the sabotage of any improvement of the individual by family members who have cast the patient in the sick role. In joint sessions with family members they are helped to accept the patient's assertiveness as a healthy development and are urged to continue supportive family relationships.

\section{References}

El-Islam, M. F. (1979) A better outlook for schizophrenics living in extended families. British Journal of Psychiatry, 135, 343-347.

El-Islam, M. F. (1994) Collaboration with families: an alternative to mental health legislation. Care in Place, 1, 256-260.

El-Islam, M. F. (1998) Clinical applications of cultural psychiatry in Arabian gulf communities. In Clinical Methods in Transcultural Psychiatry (ed. S. 0. 0 kpaku), pp. 155-170. Washington, DC: American Psychiatric Press.

El-Islam, M. F. (2001) The woman with one foot in the past. In Culture and Psychotherapy (eds W-S. Tseng \& J. Streltzer), pp. 27-41. Washington, DC: American Psychiatric Press.

El-Islam, M. F., Abu-Dagga, S. I., Malasi, T. H., et al (1986) Intergenerational conflict and psychiatric symptoms. British Journal of Psychiatry, 149, 300-306.

Kroeber, A. I. \& Kluckhohn, C. (1952) Culture: A Critical Review of Concepts and Definitions. Cambridge, MA: Harvard University Press.

\section{For contributions} to the

'Associations and collaborations' column, please contact John

Henderson, email: john.henderson53@ btopenworld.com

EFPT website: www.efpt.org

\section{Twelve years old: the European Federation of Psychiatric Trainees (EFPT)}

\section{Julian Beezhold}

President 2003-04, European Federation of Psychiatric Trainees; Crisis Resolution Home Treatment Team, Hellesdon Hospital, Drayton High Road, Norwich NR6 5BE, UK, email: julian.beezhold@nwmhp.nhs.uk

\begin{abstract}
The European Federation of Psychiatric Trainees (EFPT) is an independent federation of national trainee associations; it represents over 12000 trainees in 19 member countries across Europe. It is run by an annually elected board, comprising the President, Secretary-General, Treasurer, President elect and past President, and is governed by a written constitution.

The EFPT organises an annual forum, hosted by the current President, which is the policy-making body of the EFPT and which provides an opportunity for work on EFPT projects, academic discussion, networking and social interaction. Two official delegates represent each member country at the annual forum and each country has one vote. 0 ther trainees may attend and participate as observers.
\end{abstract}

\section{How did the EFPT start?}

In early 1992, a few members of the Collegiate Trainees' Committee (CTC) of the Royal College of Psychiatrists decided to build links between psychiatric trainees across Europe. They wrote to the embassies of all European countries to ask for information about trainee organisations. It became apparent that only three countries had a national psychiatric trainee asso ciation. However, contact was made with individual trainees in many countries and this led to an initial meeting in late 1992 in London. Sixteen trainees from nine different countries attended this informal meeting. A decision was taken to create the EFPT at an inaugural meeting in Utrecht, The N etherlands, in March 1993.

\section{Aims and objectives}

Although the aims and objectives of the EFPT have changed slightly over the years, the organisation was founded in order to do the following:

o promote representation of all psychiatric trainees

o build national trainee organisations

o provide a forum in which to learn about the diversity and richness of the current training of psychiatrists in Europe

o explore ways in which trainees can promote and improve their own training

o promulgate the opinion of the forum to relevant bodies

o promote the highest possible standards of treatment and care in psychiatry

o promote opportunities for trainees to do parts of their training, with full accreditation, in other European countries (the Exchange Programme)

O to develop policy, EFPT statements, by consensus on matters relevant to trainees.

\section{EFPT development}

The official launch ofEFPT in Utrecht in 1993 was followed by annual forums in Cork, Ireland (1994); Copenhagen, Denmark (1995); Lisbon, Portugal (1996); Athens, G reece (1997); G hent, Belgium (1998); Tampere, Finland (1999); Berlin, Germany (2000); N aples, Italy (2001); Sinaia, Romania (2002); Paris, France (2003) and Cambridge, UK (2004).

EFPT policy statements have been agreed and revised over the years on:

O general medicine and neurology in psychiatric training (1994)

O experience in research $(1994,1997,2001)$

o part-time training (1994)

o national trainees' organisations (1995)

O exchange of trainees between different countries (1995, 2001, 2002, 2003) 
Supernatural versus medical explanations of illness

Arab patients and their families may attribute behavioural symptoms to bad spirits (jinn) or attribute undesirable thoughts and wrongdoings to temptation by the devil (El-Islam, 1998). This contrasts with doctors' psychobiological attributions to stressful environmental events and mediating neurotransmitter, transporter or messenger mechanisms.

D octors should adopt a subjective ('emic') approach to understand supernatural beliefs and attitudes within a culturally shared context. If they adopt an objective ('etic') approach or an ethnocentric approach (where they employ their own culture's criteria) they not only lose rapport with their patients but also arrive at erroneous clinical conclusions.

Patients and their relatives are usually happy if traditional or religious healers collude with their projection of responsibility for disorder on to supernatural agents and include the healthy and sick in rituals aimed at neutralisation of these agents' adversity. In contrast, by attributing symptoms to the patient's own mind, doctors try to undo this projection and should be careful not to blame the patient or relatives for symptom genesis instead of supernatural agents.

\section{The patient-doctor-family triangle}

Culture makes the Arab patient-doctor relationship triangular rather than diadically linear, as there will be twoway communications between the patient, the doctor and the family. Relatives may ask for interviews with the doctor in order to provide information before the patient is seen by the doctor, or in order to obtain information after the patient is interviewed. Although most patients give doctors permission to talk to their next of kin, the latter should have no access to personal details that the patients may consider embarrassing or to information that the patient wants held in confidence by the doctor.

Tactful handling of relatives by doctors is essential for their psycho-education about psychiatric illness and for sustenance of their care for patients (El-Islam, 1979). Both relatives and patients are reluctant to reveal to doctors what they regard as family secrets, such as sexual life, history of abuse, illegal activities and details of family income (El-Islam, 2001).

\section{Collaboration with families}

Although some degree of personal independence is allowed, interpersonal concern and interdependence with minimal 'social distances' are the norm in Arabian families. The doctor is not expected to encourage adolescents to achieve Western-type full independence from their parents. $\mathrm{N}$ ot only is this culturally undesirable but there are also no socio-economic provisions for adolescents to live outside their families. Arab doctors encourage the sustenance of the interdependence group ego. The Arab family runs the affairs of its healthy and unhealthy members alike.
The decision to seek the help of professionals or traditional healers is one made by the family. In Arabian Gulf countries, the collaboration of doctors with families has made it unnecessary to introduce mental health legislation. Compulsory hospital admission of patients who do not see their need for hospitalisation is arranged with the approval of a first-degree relative on the advice of a psychiatrist. Although all patients are informed of their right to appeal against compulsory admission or detention in hospital, no such appeals were made over a 25-year period in two Arabian Gulf countries (El-Islam, 1994).

\section{Symptom appraisal}

Somatic symptoms call for the attention of doctors, who are believed to be concerned only with the body. Pains, aches and fatigue are symptoms commonly presented. Somatically oriented doctors collude with patients' somatic orientation and resort to physical examinations, investigations and treatments without exploring the psychosocial emotional factors underlying somatisation. Through excessive investigation, doctors may inadvertently promote the development of iatrogenic hypochondriasis. Their patients may believe they have mysterious or serious disease because the doctors are looking for something they cannot find.

Emotional symptoms (e.g. fears, worries or low spirits) are culturally attributed to weakness of personality or weakness of faith of affected individuals. Patients can readily accept and internalise this notion if they are depressed, and this is likely to lower their self-esteem further. Patients with predominantly somatic symptoms expect physical treatments and patients with predominantly emotional symptoms may ask for psychological treatments. Since most doctors use both medication and supportive therapy, in various proportions, they should explain that the targeted mechanisms are the same in all forms of therapy.

The third group of symptoms, overtly disturbed behaviour, is frequently attributed by patients and their families to the adverse influences of jinn. Socially disinhibited, embarrassing or aggressive behaviour, which is usually interpreted in this way, is the most shameful and stigmatising. The help of traditional healers is often sought to deal with the culprit supernatural agents. Without dismissing these culturally shared beliefs, doctors explain that medication influences the brain mechanisms of disturbed behaviour, irrespective of the factors which have caused it. The doctor should approve the patient's resort to religious methods of self-help (autoreligious therapy) rather than traditional healers' help, as the latter sometimes includes beating the patient or the administration of toxic herbs.

\section{Psychotherapy and recent developments}

Some patients who have access to information about psychiatric treatments (e.g. via the internet) are particularly
The decision to seek the help of professionals or traditional healers

is one made by the family. In Arabian Gulf countries, the collaboration of

doctors with

families has made

it unnecessary to introduce mental health legislation.

Although some degree of personal independence is allowed,

interpersonal concern and interdependence with minimal 'social distances' are the norm in Arabian families.

The doctor is not

expected to

encourage

adolescents to achieve Westerntype full independence from their parents. 